\title{
10 Looking at things
}

\author{
Delphine Mercier
}

In September 2017, while giving tours of the UCL Ethnography Collection to first-year students, I was asked by one of them - as we stood in front of a selection of artefacts - if objects were important to understanding material cultures. As a reply, I asked him to tell me about the outfit he was wearing on that day, why he decided to buy it and why he picked it up that the morning. I was hoping it would help him to understand how the relationship between people and objects is intricate and meaningful. Things matter, indeed. They are the materialisation of culture and, sometimes, objects constitute the only traces left by people. Observing the network of interactions between people and things helps us understand both a bit better, as long as we take the time to really look at them. As curator of the UCL Anthropology Department's Ethnography Collection, I spend a lot of time surrounded by, and working on and with objects. I teach students how to engage with and learn from things. In this chapter, I draw on several years' experience working with objects in the collection, critically reflecting upon the use of methodologies like object analysis or assemblage and their efficacies in the teaching and research of material culture anthropology.

Looking at things implies being physically close to them, enough to be able to touch, use, or smell them, to see all the details of their surface and of their less-accessible parts. The importance of this direct contact for a better understanding of things has been confirmed by recent neuroscience (Quiroga et al. 2011: 17) and social-science studies, several of them addressing the issue of authenticity of learning in museum contexts (Leinhardt and Crowley 2002; Hampp and Schwan 2014; Eberbach and Crowley 2005; Bunce 2016). The definition of authenticity used in these studies meets the characteristics of 'aura' by Walter Benjamin; 'the here and now of the original [that] constitutes the concept of its authenticity' (Benjamin 2010 [1939]: 13), and the uniqueness and permanence of things (ibid.: 16). As noted by Dudley, based on her experience visiting galleries (2011:2), criteria like the rarity or the age of the objects changes how we perceive them. Being aware of the authenticity of an object not only transforms the experience of engaging with it as something more valuable, but also, as Benjamin says, it puts the subject in direct contact 
with 'the quintessence of all that is transmissible' (Benjamin 2010[1939]: 14). As such, the aspect, weight, size, and smell of an object are all clues that can help us to understand the interactions between things, people, and the wider environment.

Engaging with objects is therefore a key tool in museums and collections, whether they are more traditional ones (Bunce 2016; Leinhardt and Crowley 2002) or, as with university collections, when they focus on students learning (Barnes and Lynch 2012; Vartiainen and Enkenberg 2013; Adams 2015; Kreps 2015; Krmpotich 2015). Helene Chatterjee, for example, has been doing pioneering research on the subject of objects and education, publishing multi-disciplinary research on the benefits of physical interaction with objects (Chatterjee et al. 2008) and working on objects and collections as powerful pedagogical tools for students (Chatterjee and Noble 2013; Chatterjee and Hannan 2015).

Considering that being in direct contact and engaging with original objects is a crucial way for people to understand them, the dearth of projects that focus on or start from the materiality of things is surprising. The vast majority of the sources referenced above highlight that the handling of objects is essential to understanding them. In her recent book, Museum Object Lessons for the Digital Age (Geismar 2018), Haidy Geismar details an object-based workshop, 'Properties and Social Imagination,' organised at UCL in 2012, which focuses on the materiality of the objects 'to explore the different ways of understanding and interpreting objects' (Geismar 2018: 28-62). Participants, including Geismar herself, focused on materiality, which in her case included a bark cloth, as key to understanding the objects. Physically engaging with the object is hereby not only a pedagogical tool used to illustrate ideas, but it is a method in and of itself, used to understand the object; to take 'the objects seriously in their own terms - a material-oriented perspective,' moving away 'the tendency of previous interpretive projects to incessantly convert objects into symbols' (Geismar 2018: 36-37). In this perspective, objects do not exist to illustrate ideas; they are the beginning of, and central to, the process of enquiry. In this vein, I elucidate the purpose and efficacy of an object analysis as an anthropological methodology.

\section{Object analysis}

An object analysis can be performed without having any particular knowledge of the object. The UCL Ethnography Collections is characterised - for various reasons relating to the history and provenance of the artefacts - by a lack of archives. This complicates not only the historiographic work one is able to do in and on the collection as a whole, but also with each object individually. Less than a quarter of objects in the collection have any documentation related to their provenance. This means that the information we can gather from the objects themselves is limited to the information provided by the labels, which constitutes the object's only first-hand information. As such, 
the object-analysis methodology becomes extremely important in the pursuit of understanding. This methodology, which is the focus of this chapter, is central in the teaching of anthropology at UCL. The experience of working in a collection environment with museum objects is generally new for the students. As Richard Handler describes it (Handler 1993), a collection that is a storage for objects is also a 'social arena.' Entering in the space of the collection, which has its own rules - for example, no food, no drink, specification related to objects handling - helps students to set their minds on getting ready for engaging with original objects.

As a methodology, one of the key questions of object analysis could be, paraphrasing Tilley (1994: 1): Why are particular objects made the way they are as opposed to others?' 'Phenomenology involves the understanding and description of things as they are experienced by a subject' (ibid.: 11-12) and, object analysis is based on a similar notion. Subjects enter into proximity with an object they know nothing about, with at times limited and/or wrong information. They therefore need to engage with the object using their senses, which helps them not only to find clues that they will interpret at a later stage, but also to develop an understanding of the experience of using the object. As Dudley says, sensorially exploring an object consists in 'want [ing] to touch it,' to understand 'how it would feel to stroke it, or how it would sound if I could tap the metal, or how heavy it would be if I could try to pick it up' (Dudley 2011: 1). According to Dudley, drawing on Tilley (2004) and Merleau-Ponty (1962), 'it is in the engagement between object and subject, in their very confluence, that sensory responses, emotions and ideas are generated' (Dudley 2011: 8).

As objects 'cement the ways people live together' (Lemonnier 2012: 13), the object analysis favours the development of a relationship between the subject and the object. Consequently, if the way of comprehending objects borrows from phenomenology methodology, the interpretation 'involves understanding the interconnections,' which are of a changing nature, between people, their observations and tools, the objects, the material cultures the objects belong to (Jones and Alberti 2016: 21-22). In turn, as people engage with objects as material things, their qualities become fundamental to the interpretation. At the same time, interconnections between objects, people, and their environment are also essential, as 'it is not that a material contains a finite list of inherent properties that are elicited by a knowing subject; rather, properties or qualities are the product of the interaction among subject, technology, and material' (Jones and Alberti 2016: 24 referring to Connell 2011). Thus, analysing the material, formal, structural, and functioning properties of objects can reveal localised (vernacular) relations of people with their environments, which André Leroi-Gourhan qualified as 'milieux' (Leroi-Gourhan 1971 [1943]; Leroi-Gourhan 1973[1945]; see also Coupaye, Chapter 4).

UCL Ethnography Collection ${ }^{2}$ was created by Daryll Forde, the first head of UCL's Department of Anthropology, following its establishment in 1945. 


\section{Delphine Mercier}

The collection is made of seven thousand objects and photographs, mostly collected by Forde, who stayed head of the department until his retirement in 1969 (Africa: Journal of the International African Institute 1973: 281). Forde also managed to secure donations from other collections inside and outside UCL (Petrie Collection, Wellcome Collection, British Museum, Cambridge Museum), and a long-term loan from the Cuming Collection given back in 2007 (Were 2010: 300). The objects in the department's collection come from all over the world, with emphasis on West Africa and the Australian continent, following the orientation of the research at UCL Anthropology around the time of Forde. According to Professor Michael Rowlands, ${ }^{3}$ the collection of objects was more spontaneous rather than scientifically organised, with little to no information recorded. After Forde's retirement, objects regularly joined the collection, but at a much slower pace, as less than a hundred objects have been given to the collection since the 1970s (Were 2010: 300).

Among the very few existing documents relating to the history of the collection are letters exchanged between Ashworth Underwood, director of the Wellcome Museum, and Forde between 1951 and 1954 - a time when this museum decided to re-orientate its refocus on health, the body and medicine, and to give away the rest of its gigantic collection. When he got in touch for the first time with Underwood in 1951, Forde described the collection as a teaching collection 'in connection with the teaching and research in the department.' Seventy years later, these words are still true. The collection gradually became, and still is, a valuable teaching tool, not only for various departments of UCL, but also for wider audiences including source communities and schools. Following a theoretical parenthesis (Buchli 2002a), a 'material turn' (Tilley et al. 2006) characterised by the 'rediscovery of the value of museum collections' (Adams 2015: 89) developed again from the end of the 1970s. It has been followed by a sensory turn, focusing on re-engaging with 'objects and their materiality on multisensory levels to overcome dependence on text and the purely visual' (Kreps 2015: 96-97), consequently putting 'the world of museum Anthropology and object-focused studies to a more central stage' (Adams 2015: 90). It is in this context that students learn how to engage with things using their senses and methodologies developed in the following case studies all kept in the UCL Ethnography Collection.

While the first case study exemplifies the object analysis methodology, the second and third ones illustrate the combination of object analysis and assemblage and how it allows to go a bit further in the understanding of objects.

\section{Case study one: object K.0050}

When starting an object analysis, first observations generally relate to the overall aspect of the object, such as the materials used. K.0050 (Figure 10.1 to 10.4$)^{5}$ is in a good conservational state and made of dense material. As such, it is possible not only to look at it, but also to touch, handle, and lift the object without damaging it. The object is made of one piece of wood, a dense 


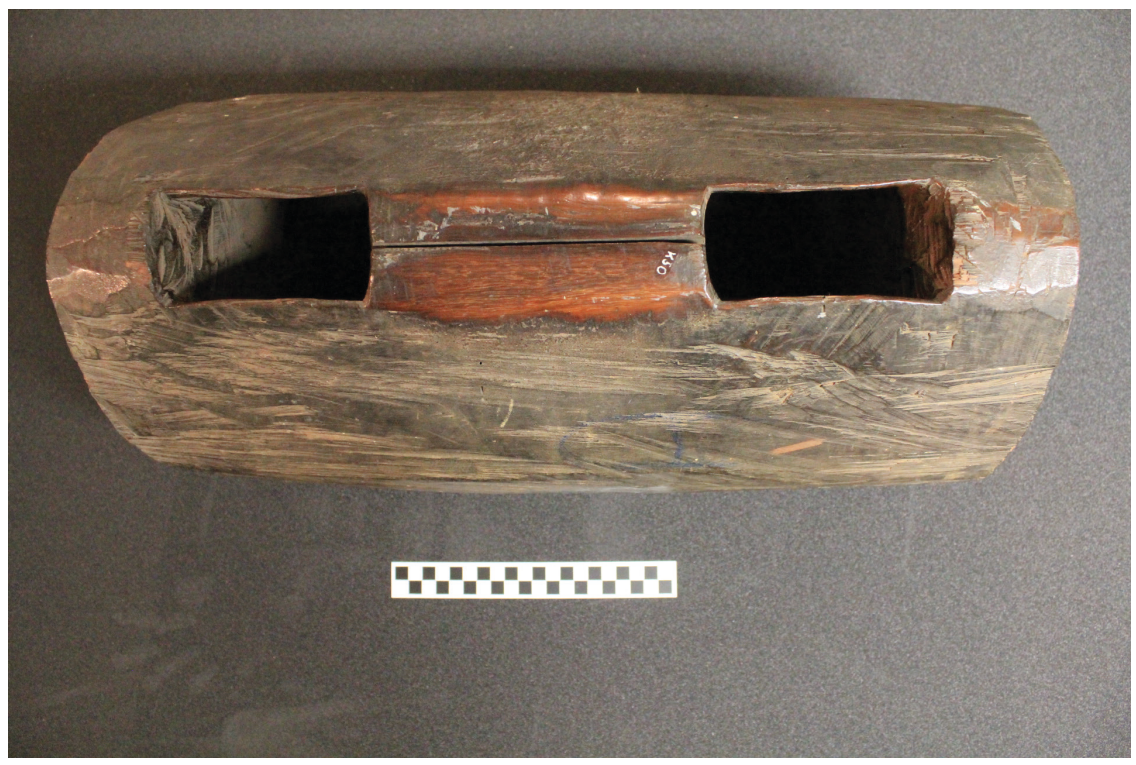

Figure 10.1 K.0050, top view.

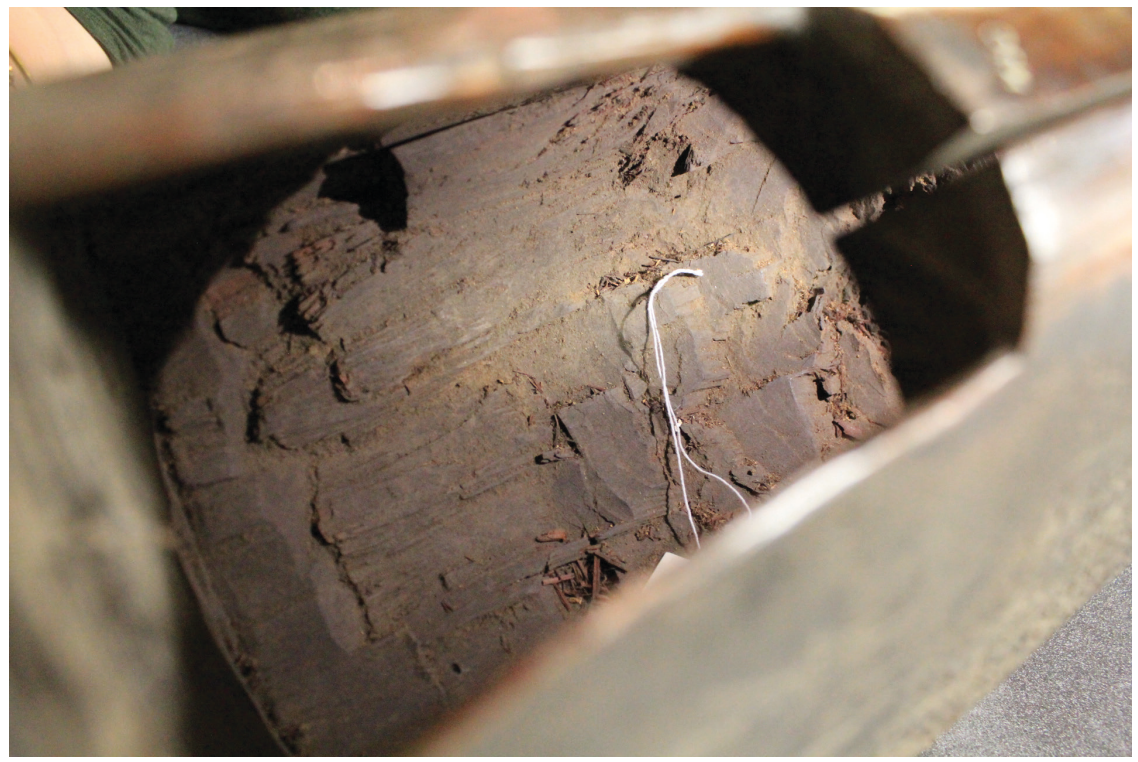

Figure 10.2 K.0050, inside view. 


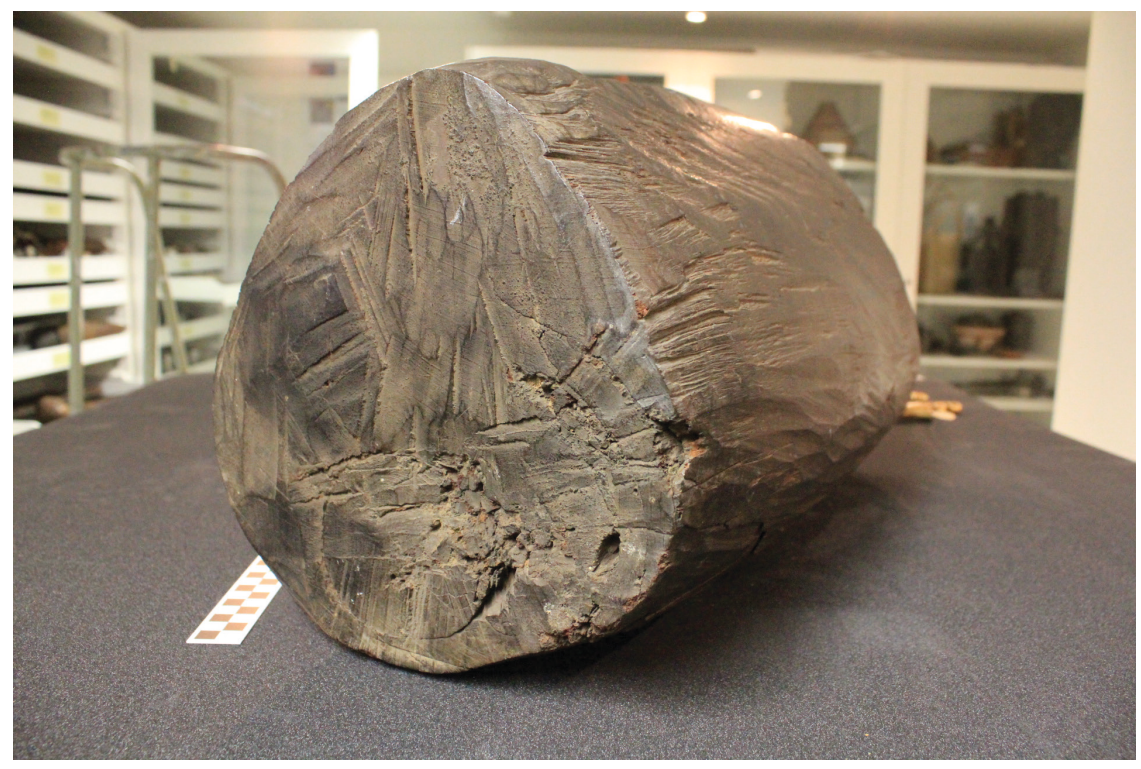

Figure 10.3 K.0050, view from one of the small sides.

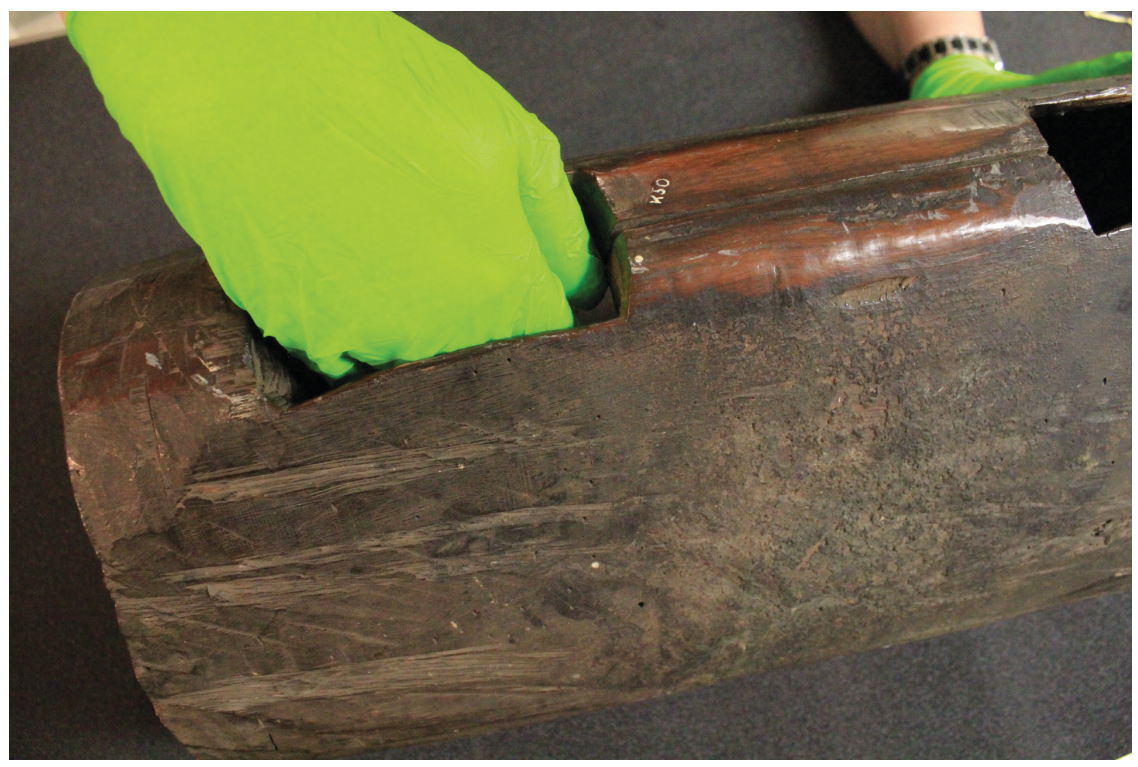

Figure 10.4 Attempt to introduce a hand inside of K.0050.

Source: Photos by Timothy Carroll CUCL Ethnography Collection. 
type, which one gathers from the weight of the object when attempting to lift it. The weight and shape of the object show that it has been made from the trunk of a large tree.

An object analysis methodology starts with sensory engagement in order to collect data that can then be analysed in a second phase. An object made of one solid large piece of tree trunk leads to the hypothesis that it was produced in an area where, indeed, there would have been trees, possibly in significant number. One could speculate that wherever K.0050 was produced, it was likely in a place where people could access or were surrounded by forests and trees of all ages. It might also indicate, or at least point, in the direction that wood might have been one of the primary local resources available, since people were able to use a sizeable part of a tree to craft one single object. This information also affords some speculation into the organisation of the material culture that produces K.0050, since the felling and transport of large trees is rarely handled by one person for safety and practical reasons. Hence, the existence of forests and the use of wood as a raw material also indicate the necessity of organised labour, or at least a need for communal work to be undertaken in order to obtain and transport the raw material to be used to create the object.

After focusing on the material itself, the next step of the object analysis focuses on how the object was made. The absence of bark on K.0050 means that it was removed before the trunk was carved. It was then carved into a pear shape (Figure 10.1). The surface of the object is covered with small incisions (Figure 10.1 to 10.3), which are two to three centimetres wide. This seems to be quite narrow for having been left on such a dense and thick material. These traces indicate that the tool used to carve the wood was made of a stronger material than the one used to make the object and had to be thin enough to leave precise and clear incisions. This could point to the use of a sharp blade of metal from the chisel type, and to the additional use of a hammer in having provided the necessary strength to leave these precise incisions. It is noticeable (Figure 10.3) that, even if these traces are mostly vertical, a lot of them have angles varying up to 90 degrees. This detail is extremely significant as it helps to mentally reproduce the gestures of the craftsperson who made this object, the movements of the body turning around the object to be able to carve it on all its sides, and the noises produced by this work.

The second part of the technical evaluation of the object would focus on the hollowing of the piece. At the top of it, there is a slit which is enlarged in a rectangular shape in the two extremities of the object. These openings (Figure 10.4) are narrow enough to make it complicated to pass a hand into them. Despite this, the inside of the trunk has been almost entirely hollowed (Figure 10.2). On one of the small sides of the object (Figure 10.3), there is a circular shape carved in the wood which is visible inside, too (Figure 10.2). This is certainly a sign that the craftsperson, after hollowing the object from the top, needed to dig a hole on the side of the object to manage to finish the work. They just had to put the piece of wood back at the end of the 
process. However, it was not only a work calling for strength: the craftsperson who made this object was also able to carve with delicacy, as shown by the very thin walls of the two top slits (Figure 10.3). The outside of the object is smoother than the inside because it has probably been roughly sanded to reduce asperities and to avoid splinters and other minor injuries. This technical evaluation of the object takes its cue from the chaine opératoire (technical sequence), both from the point of view of archaeology (Walls 2015; de Beaune 2004; Schlanger 2004), as it starts from the object, and from the perspective of anthropology (Lemonnier 2012; Coupaye 2013, 2015), as it focuses on the uniqueness of each object. The direct engagement with the object and its uniqueness offers an experience also underlined by Drazin (Chapter 5). Object analysis, via the extrapolation and associative logic of abductive thinking (see Bateson 1972, 1979), is also an open door onto the intangible parts of life, like technical movements of the body (Leroi-Gourhan 1971 [1943]) - such as hollowing and smoothing, or carving in sometimes uncomfortable positions as well as the noise of the chisel, the time invested, the smell of the wood and the shavings piling up.

After having explored this object from a technical perspective, the next stage of the object analysis would turn to exploring its uses. Except for some scratches, traces of use are visible only at the top of the object (Figures 10.1, 10.2 and 10.4) - in the middle of the long and thin slit between the two rectangular openings. Here the wood is lighter and shinier than on the rest of the surface. Even felt through a glove, the texture is extremely smooth compared with the rest of the surface. By trying to touch it with various gestures, at some point the person performing the object analysis will gently hit the surface and realise that it produces sounds, which are two musical tones as the object is a double-slit drum. ${ }^{6}$ The size of the instrument and its weight ${ }^{7}$ - trying to lift it makes one realise that it is a very heavy object - suggests that this drum was likely meant to stay on the floor, with a musician sitting in front of it. The drum produces two different tones and can therefore allow for the creation of a complex rhythm. Engagements with this drum appear to have been diverse playing it required human effort, particularly if played for a long time, while it also involves the acts of listening to it, and possibly also dancing to and around it. Other questions could also emerge, such as when was the drum played, during the day or the night? This aspect provides wider insight into the sound of the environment, in the context wherein a material object produces specific types of non-monotonal and rhythmical sound. Finally, as this object is a musical instrument, the craftsperson who made it had to be aware of the musical properties of the wood, knowing exactly how much to hollow it, or how thin the walls of the opening needed to be to produce the desired effects perhaps the craftsperson and the musician had been the same person.

As shown in this example, an object analysis does not allow for a complete understanding of the entirety of the object. Without the adjoining label, information like the country of origin of the object for instance would stay unknown. It is also not possible to generalise all the interpretations made 
on one object as each object is unique, and even if it has not been handmade but machine made and mass-produced, the uses of objects differ, in discrete contexts. Therefore, in analysing an object, as in engaging with them in an exhibition as exemplified by Jeevendrampillai, Burton, and Sanglante (Chapter 7), it is necessary to understand the relationships between object, people, and environment, such as: the interactions existing between the makers of the object and the object itself, the object and the people who used it, the makers and the users, including the dynamics between them, as well as the situated context of the observer (the person performing the object analysis).

A thorough understanding of these networks in as holistic a way as possible is important in performing an object analysis on one isolated object. However, an object is rarely completely isolated and, in the case of a collection for instance, it might at least be related to the rest of the collection. By using the archaeological methodology of assemblage in a collection, it is possible to create connections between objects. This method is defined by Joyce and Pollard (2010: 292) as 'a collection of materials related through contextual proximity [making] possible to interpret the group of materials as evidence for specific events, processes, or practices in the past.' The assemblages, whose 'properties emerge from the interactions between parts' (Manuel DeLanda in Bille and Sørensen 2016: 16), also constitutes a first attempt of a stratigraphy of the collection. As Jones and Alberti argue (2016: 29), 'rather than interpreting the meaning of the artefacts they excavate through contextual analysis, archaeologists shape and compose the assemblages that they excavate; through this process of composition, interpretation and evaluation arises.' They conclude by stating that 'we have shifted from a conception of archaeological interpretation as a largely cognitive or cerebral endeavour to a recognition of the physical and material aspects of archaeological interpretation.' This dynamic relationship between the cognitive and the material leads to a better understanding of both. This is reminiscent of Bateson's theory of double-description (Bateson 1979: 132), whereby the observational description of an object and the abductive inferences about the object produce a deeper understanding of the object at hand based on the relationships of resemblance and difference between the object and those previously known to the observer (see also Küchler, Chapter 9 and Küchler and Carroll 2021b). Thus, in coming to 'look at' or 'be with' an object in an analysis, new understandings arise with 'reference to a particular pattern of interaction.'

Following on the dynamics of Bateson's double-description, case studies two and three use assemblage method - based on comparisons, interactions and inference - for a better understanding of objects and their context of making.

\section{Case study two: the Inuit collection}

At this point, I examine the significance of the environment in understanding objects through assemblage. In the following case study, assemblage 


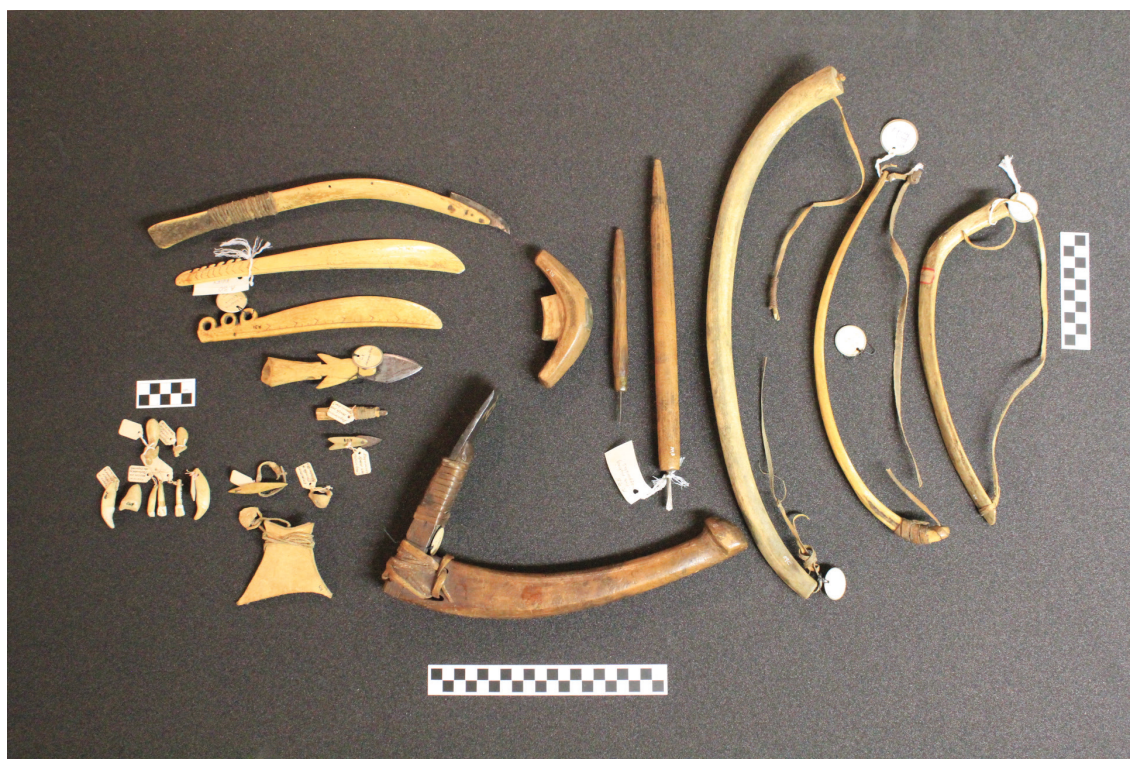

Figure 10.5 The whole Inuit collection kept in UCL Ethnography Collection.

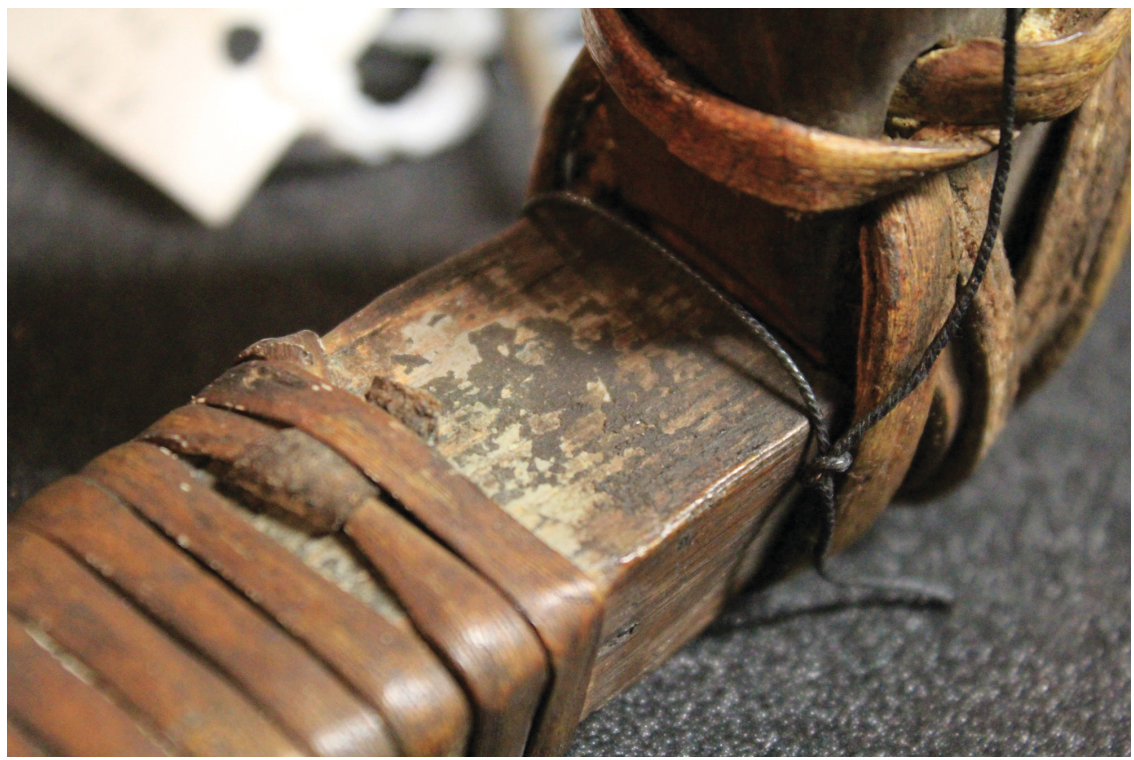

Figure 10.6 Detailed view of the adze, showing the different layers of paint used to cover the wood every time it was reused.

Source: Photos by Timothy Carroll CUCL Ethnography Collection. 
methodology is used to understand the environment, in particular relation to the resources available and the interactions between resources and people.

The UCL Ethnography Collection keeps 24 objects from the Inuit/Inupiaq/ Yupik/Eskimo ${ }^{8}$ area. ${ }^{9}$ By assembling and engaging with these items, we will try to understand how we can at least partially understand the environment in which they have been created and, consequently, the kind of techniques and knowledge needed locally to make things. The methodology of assemblage has been already used in the context of museums, notably to focus on issues like provenance and context (Flexner 2016). Here, empirical observation and sensory engagement will be used to highlight materials in relation to resources that were locally available at the time of the objects' production.

The Inuit collection is comprised of: one whittling knife; bow-drill tools consisting of two drills, three bows and a mouthpiece; a collection of five perforated animal teeth; a non-perforate animal tooth; two pieces on the tingmiujang game (Martjin 1964: 557); two yaaruin or story knives; a spearhead; a knife; a harpoon head; sewing equipment consisting of one buckle, two thimbles, a needle and a hand protection; an adze.

Table 10.1 Materials used to make the objects of the Inuit collection

\begin{tabular}{lll}
\hline Materials & $\begin{array}{l}\text { Number of objects in which } \\
\text { the material is used on a } \\
\text { total of 24 objects }\end{array}$ & Raw materials \\
\hline Animal bone & 7 & Animal \\
Animal skin & 8 & Animal \\
Musk Ox tooth & 3 & Animal \\
Wolf tooth & 2 & Animal \\
Animal tooth & 1 & Animal \\
Walrus ivory & 5 & Animal \\
Reindeer sinews & 1 & Animal \\
Reindeer skin & 1 & Animal \\
Wood & 5 & Vegetal \\
Fibres & 4 & Vegetal \\
Iron & 6 & Mineral \\
Native iron (?) & 1 & Mineral \\
Meteoric iron (?) & 1 & Mineral \\
Stone & 1 & Mineral \\
\hline
\end{tabular}

Table 10.2 Raw materials present in the Inuit collection

\begin{tabular}{lll}
\hline Raw material & Occurrences & Different types of material \\
\hline Animal & 28 & 8 \\
Vegetal & 9 & 2 \\
Mineral & 8 & 4 \\
\hline
\end{tabular}


The two tables above detail the composition of the Inuit collection objects. It shows a list of simple observations I undertook in the collection, as seen in Figure 10.6, from which one is able to draw some conclusions:

- Animal materials are the most frequently used materials among these objects;

- All the animal materials come from mammals;

- The parts of animals used are diverse;

- The minerals used are diverse;

- Vegetal resources are minimal.

These considerations on materials are interesting primarily because they give us an insight into the environment in which these objects were made. As ethnonyms, 'Inuit,' 'Inupiaq,' 'Yupik'or 'Eskimo' refer to several related people groups living across a very large area marked by long and cold winters and short and cool summers (subarctic climate). Within the assembled objects, and as shown in Table 10.1, a significant number of mammals and a variety of body parts have been used in the manufacturing of the objects. ${ }^{10}$ Whether partially farmed like reindeer or wild like walrus, these mammals have been killed for at least two reasons in the context of our case study: to be a source of energy for their meat and fat; and/or to be a source of raw material, namely their bones, skin, sinews, and teeth. That these animals have been used in a variety of ways indicates that they are likely one of the most readily available sources of material locally. This would, for instance, explain the use of a mammal rib to make the bow-drill: a branch would have been much easier to use, just needing to be cut - instead of killing and butchering an animal before one could cut the rib and clean it. The quantity of animal parts used, in the context of the Inuit collection, strongly suggests that animals were among the main materials available locally in this context.

At the other end of the spectrum of available raw materials, there is metal. Only the inscription on the label allows us to identify the nature of the meteoric iron (Buchwald and Mosdal 1985) without any further analysis. Concerning the bullet, its size and shape enable its identification. At the top of the object, there is an inscription following the circular shape of the bullet, W.R.A.CO. on the upper part, and .44 W.C.F. on the lower part. Winchester Repeating Arms Company, Caliber $44 \mathrm{WCF}$ were produced from 1873 to 2011, and Winchester used the 'W.R.A.CO' head-stamp until 1928 (Adkins 2011). The origins of the metal parts are diverse, which indicates that people tried to find metal wherever they could. Additionally, apart from the adze, the bits of metal represent only a small percentage of each object and are used only where they cannot be replaced by another material. A piece of stone replaces, for instance, what could have been made in metal in the mouthpiece of the bow-drill. This could be a sign of the metal's limited local availability, and, consequently, this could signify the ability of Inuit people to find, use, and technically work with metals of different origins, thereby obtaining the best from their environment. 
Materials of vegetal origin such as fibres and wood are commonly used by Inuit people, but in fewer varieties. Considering the specificity of the weather in the Inuit area, fibres could only be collected during the summer, which shows the importance of the warmer season regarding elements such as food diversity and the gathering of raw materials to survive the rest of the year. Wood is not a commonly known resource in Inuit areas. It is therefore interesting to notice how it has been used for objects or parts of objects, whereby the physical qualities of wood were needed to enable lighter, but solid, tools. When the qualities of wood were not needed, other more common materials would have been used. In the case of the bowdrills, the elasticity of wood might have facilitated their use and conservation as would have fibres, instead of using ribs and skins. The two later did not possess all the qualities of the vegetal materials but enough to fulfil the requirements of the object. They were therefore favoured, as they were easier to obtain.

With the noticeable exception of the adze, wooden elements do not feature much among the objects of the collection, which is another indication of the parsimonious use of this material. A sizeable amount of wood was used to make the handle of the adze, which is the result of very skilled work: the clear and precise shape, including its being slightly curved, as well as the finishing, all reveals the skills of the craftsperson who made it. Considering the quality of this work, it would have been simple for the craftsperson to carve the whole handle from one piece of wood, which technically also would have made it more solid and efficient. Instead, the craftsperson has used two different pieces of wood, both rather light. This reflects practical considerations, such as being lightweight to carry, while it also made the object less solid overall. The craftsman has tied these pieces together, using what appears to be leather cordage made of some kind of animal skin. A possible reason why the craftsman opted for this solution might have been the absence of a piece of wood that was sufficiently large, long, and thin enough to work with. By giving close attention to the piece of wood attached to the metal blade, it is possible to note traces of paints, in two layers, one light and one dark. These traces are visible below the skin cordage that holds the adze together. There is no paint in the most accessible part of this piece of wood, probably because it was sanded. The visible traces of paint signify that this piece of wood was used previously in another way before being transformed into an adze. In its previous uses, it has been painted at least twice, and recycled at least once - if not more often. Considering the lack of trees in the Inuit area, the most common sources of wood were indeed driftwood and second-hand wood (Alix 2012).

In this case study, assemblage has been used to reach a better understanding of the environment in which people were living and, consequently, the materials available to them and the techniques they needed to master in order to work these materials. Our last case study shows how the methods explained in this chapter can provide a framework for developing speculation that may serve as a hypothesis to test in future lines of investigation. 


\section{Case study three: the Fijian collection}

The Fijian collection of UCL Anthropology consists of 18 tapas, a tapas beater, ceramics, and weapons. The main raw material used to make these objects is wood. The amount of wood needed to make all of these different

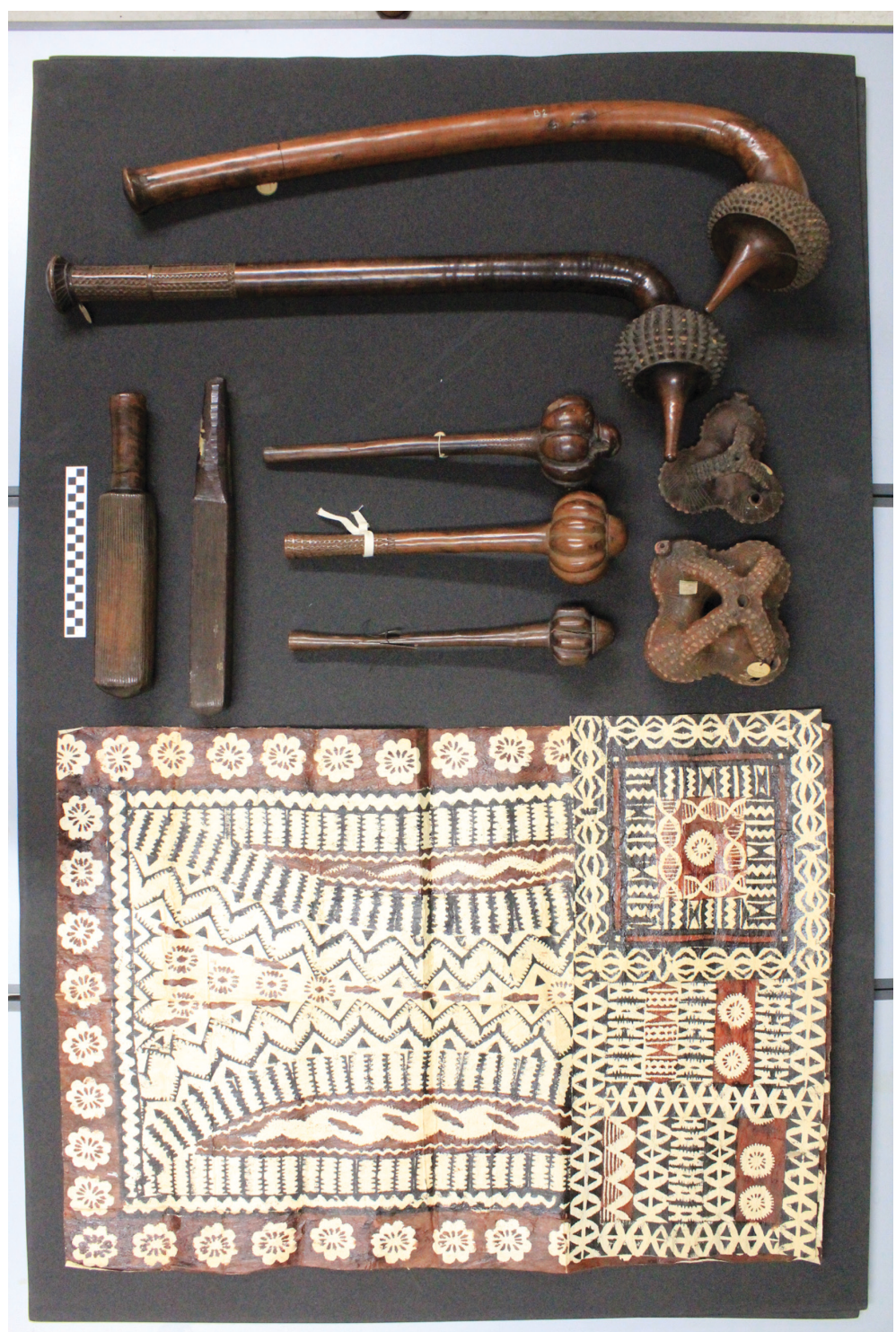

Figure 10.7 The major part of the Fijian collection kept in the UCL Ethnography Collection.

Source: Photo by Timothy Carroll CUCL Ethnography Collection. 
objects indicates, as per our first case study K.0050, an abundance of trees and, consequently, forests. Looking specifically at these objects, it is important to try and ascertain which pieces of tree have been used for which item. All the tapas are made of bark, and stencils were used to make the visible patterns. The stencils were just tools, which were not meant to be displayed but only to be used. These needed to be easy to carve with and use, and cheap to produce, in a material supple enough to be used several times without breaking and without taking the pigments in. Leaves would certainly fulfil these criteria. As for the pigments, they were traditionally 'obtained from plants, trees and earth pigments' (Flowers et al. 2019) and applied, as traces visible on tapas show, either with fingers or with some fibres, grass or thin pieces of wood when more precise design was needed. Tapas are obtained by beating bark and, when it is related to their uses, they are the equivalent to any other textiles in material culture practising weaving. Therefore, they are mostly used to ornate and protect the body.

The rest of the objects in this assemblage, apart from the ceramics, are each made of one piece of solid and heavy wood. Clubs are interesting as they follow the natural shape of roots or branches, their heads being carved in the nodes formed at junctions of elements. This is the reason why the straight parts of clubs are not entirely straight as they just follow the natural shape of branches. Therefore, these clubs are incomparably more solid than if they were made of two different parts put together. These clubs are weapons; the small ones are projectiles, and the large ones for contact at close range. Considering their weight, it was probably much easier for men rather than for women to hold and use them.

In this case study, trees are almost the only source of raw materials used. As trees were used extensively, we can assume that they were locally abundant. Therefore, people developed various techniques to utilise different parts of the materials to hand. At a wider level, it could be interesting to speculate that this might mean that trees could occupy a more symbolic place amongst these people. It is difficult to say, particularly from the object analysis alone, but if we follow this idea and try to picture the local society in a wider context, it might allow for new ways to drive ethnographic investigation. For example, does the source location of the various types of wood correlate with the social role of the people using these items? The tapas, made from the bark of the trees, wraps the body's 'trunk'; the branches, rendered into weapons, are used by warriors to extend their own 'branches' (i.e., arms) at length. While the object analysis and assemblage do not allow the analyst to go further than abductive suppositions, these methodologies of engagement make a strong case for understanding the relations among materials, techniques, objects, peoples, and society and point to its organisation at a wider, symbolic level.

\section{Conclusion}

Looking at things in a museum or archaeological context via a phenomenological approach starts from a place of engaging with things. As explained in 


\section{Delphine Mercier}

this chapter, looking at things is used here in its mainstream definition. When we say we are looking at a piece of cake at the bakery before choosing it, we are using a range of senses in addition to visual. Similarly, with the object analysis, we are engaging with the thing using our bodies. What we commonly mean by looking in this context is thus actually wider than just seeing and, in the case of the object analysis, it is clear that the engagement of almost all the senses involves being with things rather than only looking at them. Objects are highly experiential, but being with things is not simply a subjective process - there are a series of deductions and analyses one can draw from the object itself, as shown. Paraphrasing Don Ihde, who argues that science is embodied through its instruments, we can say that technique, tools, knowledge, and knowhow are embodied in the traces they leave on objects (Ihde 2008: 7). For Ihde, the idea of interaction is key, as it is for object analysis, or for assemblage, whereby he insists on 'Humans and technologies' being 'interrelational and mutually co-constitutive' (Ihde 2010: 135). Therefore, unlike other pedagogical methodologies developed in relation to engaging with collections, being with things using object analysis and assemblage as examined in this chapter, is not only significant due to the investigative-analytical experience itself, but also because it is an effective way for non-specialists to understand objects and people in relation with all kinds of objects. Being with a thing, in other words, continues to be a powerful way of understanding material cultures.

\section{Acknowledgements}

I would like to thank Timothy Carroll and Shireen Walton for their attentive and kindly re-reading, which helped me to enlarge the critical frame of this study.

\section{Notes}

1 Tilley 1994: 1: 'The key question addressed is deceptively simple: Why were particular locations chosen for habitation and the erection of monuments as opposed to others?'

2 UCL Ethnography Collection belongs to UCL Anthropology.

3 Interviewed by Delphine Mercier in UCL Ethnography Collection on 18 November 2019.

4 Objects are more than numbers, but I voluntarily use here the dry appellation at the beginning of the object analysis, as I wish to emphasise the path of the relationship between the observer and the object, which becomes more intimate at the end of the object analysis.

5 The label of the object indicates that K.0050 has been collected in Nigeria among the Efik people. The UCL Collection does not have a record of how it was collected nor when it entered the collection.

6 This object is actually not a drum, but an idiophone, as its sound is created by the vibration of the wood itself, not by the vibration of another added material like a membrane. 
7 UCL Ethnography Collection is unfortunately not equipped with scales with a range large enough for this type of object, so it is not possible to provide a precise number for the weight of K.0050.

8 On the use of Inuit and Eskimo, see Mailhot 1978 and Kaplan n.d.

9 UCL Ethnography Collection 24 Inuit objects: A.0030, A.0031, A.0032, A.0108, A.0109, C.0008, C.0036, E.0007, J.0108, M.0018, M.0019, R.0008, R.0009, R.0010, R.0071, R.0072.

10 On the articulation of vital and technical processes, see Coupaye, Pitrou, and Provost 2016. 